\title{
Linear Polarization Towards Galactic Anticenter direction: the Case of Alessi 1
}

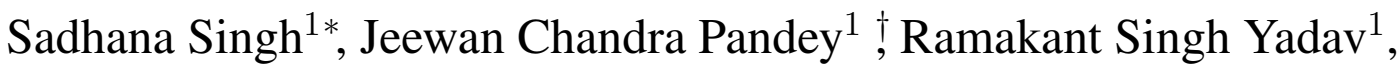 \\ Biman Jyoti Medhi ${ }^{2}$, Arti Joshi ${ }^{1}$, Bharti Arora ${ }^{1}$ \\ ${ }^{1}$ Aryabhatta Research Institute of Observational Sciences (ARIES), Manora Peak, \\ Nainital 263001, India \\ ${ }^{2}$ Gauhati University, Guwahati 781014, India
}

\begin{abstract}
We present linear polarization towards the direction of the star cluster Alessi 1, which is located in the anti-galactic centre direction. We have observed the cluster using ARIES IMaging POLarimeter (AIMPOL) as a back-end instrument at 104-cm Sampuranand telescope, ARIES, Nainital. The average degree of polarization towards the cluster Alessi 1 are found to be $0.80 \pm 0.38 \%, 0.80 \pm 0.23 \%, 0.81 \pm 0.26 \%, 0.86 \pm 0.32 \%$, in B, V, $\mathrm{R}$, and I photometric bands, respectively, whereas the position angles are found to be $86 \pm 11^{\circ}, 81 \pm 10^{\circ}, 78 \pm 8^{\circ}$, $85 \pm 8^{\circ}$ in B, V, R, and I bands, respectively. Polarization towards Alessi 1 cluster is dominated by foreground dust grains and probably a single layer of the polarized source appears to exist at $\sim 0.7 \mathrm{kpc}$ close to Alessi 1 cluster. The minor axis of dust grains towards the cluster Alessi 1 appears aligned along the galactic magnetic field.
\end{abstract}

Keywords: polarization - cluster - cluster: individual (Alessi 1) - dust - interstellar matter

\section{Introduction}

Studies of polarization due to the interstellar matter (ISM) are important as these provide information about the properties of the dust associated with the ISM and intra-cluster matter as well as help to trace the Galactic magnetic field. According to Davis \& Greenstein (1951), the polarization of starlight is caused by the selective extinction due to the elongated dust grains aligned in space possibly due to the magnetic field. For this, dust grains must have sizes close to the wavelength of incident radiation. As the grains are thought to align due to the local magnetic field, the observed polarization vectors map the general geometry of the magnetic field. Our main aim is to investigate the properties and distribution of dust grains towards the anti-galactic center direction. For this purpose, we have selected open star clusters at different locations towards the Perseus arm of the Galaxy. The polarimetric study of open star clusters provides valuable information about foreground interstellar dust, because of the available knowledge of their physical parameters such as distance, membership probability and colour excess.

\footnotetext{
*email:ssingh@aries.res.in

†email: jeewan@aries.res.in
} 
For the current study, we have selected an open star cluster Alessi $1\left(1=123.26^{\circ}, \mathrm{b}=-13.30^{\circ}\right)$. According to Alessi et al. (2003), Alessi 1 has a large diameter of 54' with an isolated group of stars. The pieces of evidence they have given for Alessi 1 to be a cluster were a fairly well Digitized Sky Survey image with the presence of significant features in the Radial Density Profile and Vector Point Diagram diagrams. They found 28 probable members of the cluster using the kinematic analysis. We present preliminary polarimetric results from this open star cluster.

\section{Observations and Data Reduction}

The optical polarimetric observations of the cluster Alessi 1 were performed from AIMPOL mounted at the Cassegrain focus of the 104-cm Sampuranand telescope of ARIES, Nainital. The telescope is a Richey-Chreteian reflector with focal ratio $\mathrm{f} / 13$. The detector is a Tek $1 \mathrm{k} \times 1 \mathrm{k}$ Charge-coupled device camera cooled by liquid nitrogen. Details about the polarimeter and its data reduction are described in Rautela et al. (2004). Observations were taken at four positions of half wave plate $\left(0^{\circ}, 22.5^{\circ}, 45^{\circ}, 67.5^{\circ}\right.$ from North-South direction). A diameter of $24^{\prime}$ of the cluster region along with polarized (HD19820) and unpolarized (HD21447) standard stars were observed on 22 and 23 November 2017 with exposure time of 180, 120, 25, and 50 seconds in B, V, R, and I photometric pass-bands, respectively. Image Reduction and Analysis Facility astronomical software was used for performing aperture photometry. Correction for instrumental polarization and zero point calibration of position angle were done using unpolarized and polarized standard stars observed on the same night.

\section{Results and Discussion}

The degree of polarization and position angle of stars in the cluster region were extracted in $\mathrm{B}, \mathrm{V}$, $\mathrm{R}$, and I photometric passbands. Overlapping of an ordinary image with the adjacent extraordinary image cannot be avoided due to the absence of grid in AIMPOL. Therefore, in the care of target sources, we have selected only those isolated stars which do not show any overlapping of ordinary image with adjacent extraordinary image. This turned to be only 79 stars in the observed field of view of the cluster Alessi 1. The distribution of degree of polarization of these stars in the $\mathrm{R}$ band is shown in Fig. 1.

Mean values of polarization and position angle in different bands are calculated by fitting the Gaussian curve in their distribution and are given in Table 1. While calculating the mean value of polarization and position angle, we have included all members and field stars of the cluster. The

Table 1: Mean value of polarization and position angle for stars in Alessi 1.

\begin{tabular}{lcr}
\hline Passband & $\mathrm{P}(\%)$ & $\theta\left(^{\circ}\right)$ \\
\hline $\mathrm{B}$ & $0.80 \pm 0.38$ & $86 \pm 11$ \\
$\mathrm{~V}$ & $0.80 \pm 0.23$ & $81 \pm 10$ \\
$\mathrm{R}$ & $0.81 \pm 0.26$ & $78 \pm 8$ \\
$\mathrm{I}$ & $0.86 \pm 0.32$ & $85 \pm 8$ \\
\hline
\end{tabular}

degree of polarization of a star depends on the column density of aligned dust grains that lie in front of the star, if the star is not intrinsically polarized. For the member stars of the cluster, the degree of polarization would be same unless a star is intrinsically polarized. Hence polarization may constrain on the membership of the cluster. A plot between Stokes parameters Q and U, also known as Stokes plane, is a first step to separate the members and non-members in the cluster region. The 


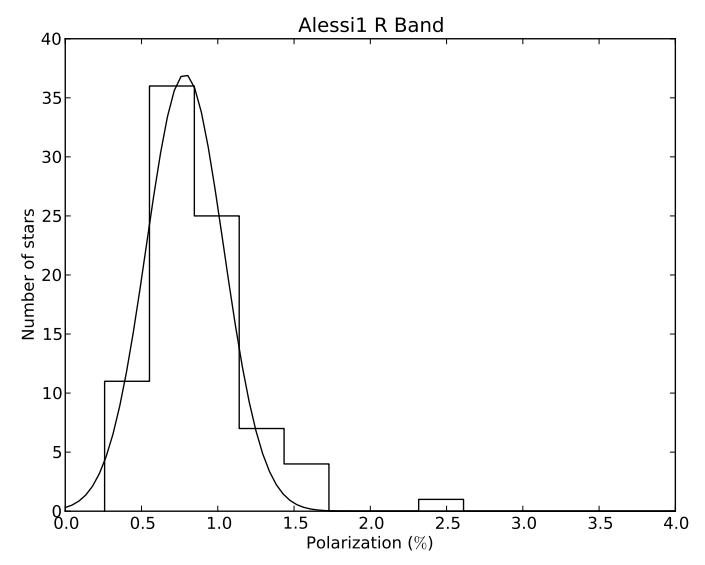

Figure 1: Distribution of degree of polarization among observed stars in $\mathrm{R}$ band is shown. The continuous curve shows the best fitted Gaussian curve.

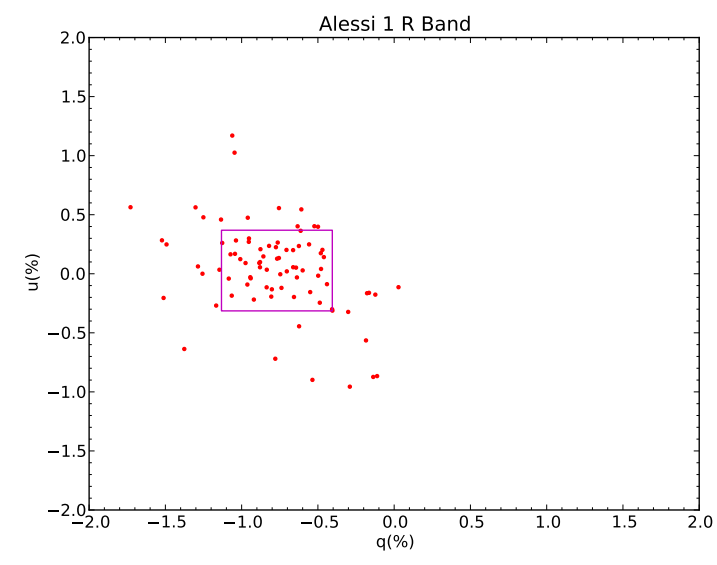

Figure 2: Plot between normalized Stokes parameters for observed stars in $\mathrm{R}$ band. $1 \sigma$ box is drawn using the mean and standard deviation of Stokes parameters $q$ and $u$.

members of the cluster are expected to show a grouping in the plot and non-members to show scattered distribution. Hence, to get the preliminary information about membership of the cluster Alessi 1, we have plotted normalized Stokes parameters $\mathrm{q}$ and $\mathrm{u}$ in $\mathrm{R}$ band, which is shown in Fig. 2. Stars which are outside the $1 \sigma$ box may not be the member of the cluster, whereas the stars inside the $1 \sigma$ box could be probable members of the cluster. We found a total of 52 stars inside the $1 \sigma$ box. Several sources inside the $1 \sigma$ box may be non-member as polarization not only caused by the scattering of dust in front of the cluster region.

Using the Vector Point Diagram (VPD) and Colour-Magnitude Diagram (CMD) with Gaia Gband brightness $\leq 20 \mathrm{mag}$, we have identified 34 stars to be members of the cluster Alessi 1. VPD and CMD diagrams were generated using the data obtained from the Gaia mission (Lindegren et al. 2018). Out of these 34 stars, 17 stars were observed for the polarization and lied within the $1 \sigma$ box as described above. These 17 stars share common proper motion and similar polarization values, which indicate that these stars are confirmed members of the cluster Alessi 1. Though this number is only around $61 \%$ of the member identified by Alessi et al. (2003). This could be due to a longer field of view taken by Alessi et al. (2003).

In order to get the information about foreground dust concentration, the variation of linear polarization with distance is important. The degree of polarization increases with the distance of stars because of a large column of aligned dust grains. If the radiation from the star encounters from the dust layer at a distance then the value of polarization will show a sudden jump with the distance. Hence the number of dust layers encountered by radiation of star along the path can be calculated by the number of sudden jumps in polarization value. We have plotted polarization with distance for $\mathrm{R}$ band in Fig. 3. The distance of each star is taken from Gaia (Lindegren et al. 2018).

The constant polarization with distance indicates that there is a single layer of the polarized source close to $0.7 \mathrm{kpc}$ towards the direction of Alessi 1.

\subsection{Interstellar Polarization}

Wavelength dependence of polarization can be used to constrain the dust properties. It follows the following relation given by Serkowski et al. (1975).

$$
P_{\lambda}=P_{\max } \times \exp \left[-K \times \ln ^{2}\left(\lambda_{\max } / \lambda\right)\right]
$$




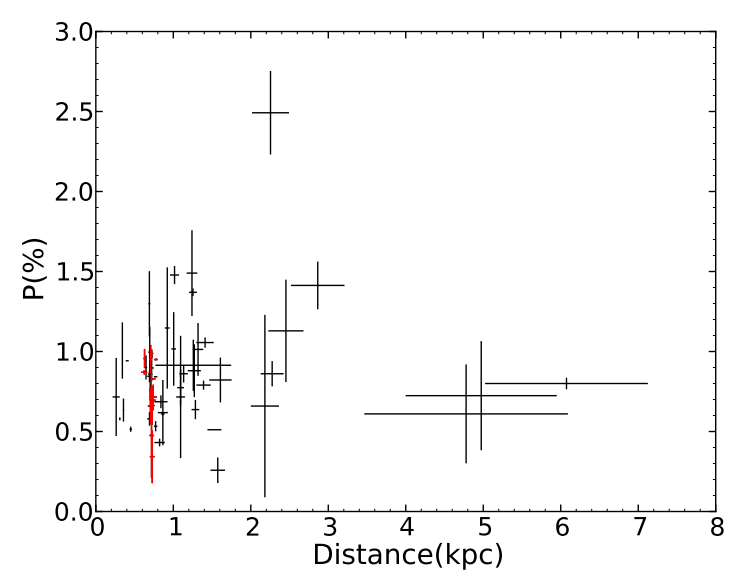

Figure 3: Variation of polarization with distance in $\mathrm{R}$ band. Confirmed member stars are shown in red colour.

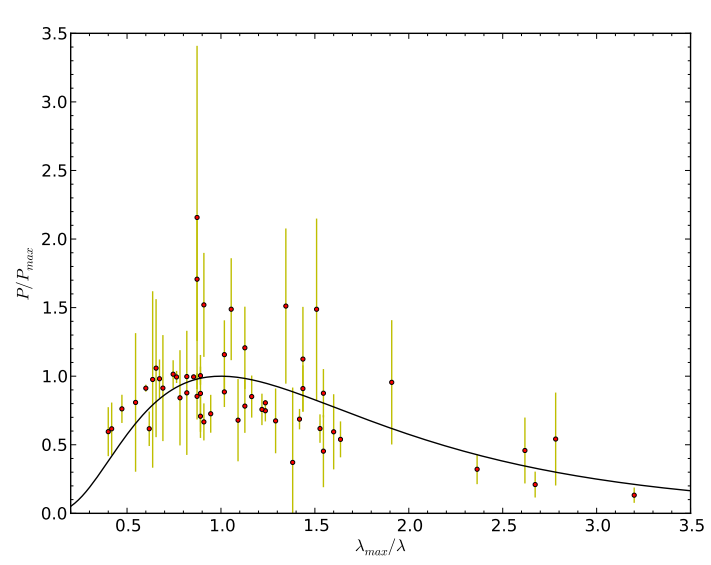

Figure 4: Normalized plot for variation of polarization with wavelength.

where $P_{\lambda}$ is the polarization value measured at wavelength $\lambda$ and $\lambda_{\max }$ is the wavelength at which maximum polarization $P_{\max }$ occurs. $\lambda_{\max }$ is a function of the optical properties and characteristic particle size distribution of aligned grains and have values from $0.45 \mu \mathrm{m}$ to $0.8 \mu \mathrm{m}$ (Serkowski et al. 1975). $\mathrm{K}$ is inverse of the width of the polarization curve and is taken as 1.15 (Serkowski et al. 1975). The criteria for ISM polarization is well represented by the value of $\sigma_{1}$, which is the unit weight error of fit. If $\sigma_{1} \leq 1.6$, then the polarization is supposed to be due to the interstellar medium i.e. the wavelength dependent polarizations are well explained by the relation 1 . However, $\sigma_{1}>1.6$ indicate intrinsic polarization. Considering only members of the cluster, we got weighted mean of $\lambda_{\max }$ and $P_{\max }$ to be $0.68 \pm 0.08 \mu \mathrm{m}$ and $1.11 \pm 0.09 \%$, respectively. The normalized Serkowski empirical curve for all stars is shown in Fig. 4. The black solid curve is Serkowski polarization relation for general ISM. The wavelength $(\lambda)$ is fixed at the average value of $\lambda_{\max }$ for the general interstellar medium of $0.55 \mu \mathrm{m}$. In this plot, there exists a good agreement between theory and observation. Polarization for stars is well represented by Serkowski law. We can conclude that polarization in the cluster Alessi 1 is due to the interstellar medium dust grains.

\section{Conclusions}

We have observed linear polarization for 79 isolated stars in the region of Alessi 1 cluster. Polarization towards Alessi 1 cluster is dominated by foreground dust grains, where a single layer of the polarized source appears to exist at $\sim 0.7 \mathrm{kpc}$ close to Alessi 1 cluster. The weighted mean of $\lambda_{\max }$ and $P_{\max }$ are found to be $0.68 \pm 0.08 \mu \mathrm{m}$ and $1.11 \pm 0.09 \%$, respectively. Dust grains are mostly aligned by galactic magnetic field towards the direction of the cluster Alessi 1.

\section{References}

Alessi B. S., Moitinho A., Dias W. S. 2003, A\&A, 410, 565

Davis L. J., Greenstein J. L. 1951, ApJ, 114, 206

Lindegren L., Hernndez J., Bombrun A. et al. 2018, A\&A, 616, A2 (Gaia 2 SI)

Rautela B. S., Joshi G. C., Pandey J. C. 2004, BASI, 32, 159

Serkowski K., Mathewson D. L., Ford V. L. 1975, ApJ, 196, 261 